\title{
18. INFORMATION ORGANIZING AND SHARING TECHNIQUE FOR TASK PERFORMANCE AND TRAINING SUPPORT
}

\author{
Hiroyuki Kojima, Takaaki Yamada, \\ Yoshihiro Mizuno, Toshiyuki Yuasa \\ Systems Development Laboratory, Hitachi, Ltd. \\ 8-3-45 Nankouhigashi, Suminoeku, \\ Osaka, 559-8515 Japan \\ Phone:+81-6-616-1107 Fax:+81-6-6161109 \\ e-mail:kojima@sdl.hitachi.co.jp
}

\begin{abstract}
There is needed a computer system that quickly transfers business procedures, task knowledge that relates to complicated business training or instruction terms under networked databases environment. We describe the human interface for effective retrieval interaction such as a task adaptive electronic manual for filling out a form. For the distributed task information available on the internet or on intranet in the product design field, we propose information organizing and sharing method as a means of getting timely and exact knowledge for a task performance.
\end{abstract}

\section{Keywords}

Higher Education, Human Computer Interface, Information Retrieval, Networks 


\section{Introduction}

Technological innovations and a changing economic structure are forming a society in which knowledge dominates the core part of production capacity and requires workers to optimize their productivity (Nonaka,1995). They are confronted with this requirement as it relates to work using knowledge. Therefore, in every business field, user support technologies are expected to aid and support the activity of workers. Support elements such as advisory, librarian ship, reference, and training function must sufficiently deal with the problems that workers face (Bramer, 1994). In the past, the functions of these elements were put together in the form of a manual. The electronic manual is an OJT (on the job training) oriented performance model that combines increased business performance and business instruction/training (Kojima,1992 Haga,1993 Yamada,1997). It should essentially satisfies the requirements of high understanding of a task procedure, supplying knowledge and know-how at the task execution site, and acting as a task instructor and a task manager.

On the other hand, workers must get timely and exact information for their task performance because this information may frequently change according to rapid progress in economic structures and network technology. So an information transfer method, in this case a dynamic electronic manual, must be able to cope with the above mentioned changes, instead of a typical business manual and execution manner which is systematized and arranged from a long historical standpoint. Basic technology in which workers can change the situation, viewpoint and view angle as well as extract useful parts, change, and add comments to the information is expected. So it must contain an interface that properly presents information for business performance to each person and have information transfer and acquisition mechanism that is oriented toward performance improvement.

In this paper, we first study user interfaces for effective retrieval interaction such as a task adaptive electronic manual for concurrently realizing business performance and training for a solution to the above mentioned problem. Second, we propose the information organizing and sharing method, composed of information watching and gathering, organizing and viewpoint sharing. This conception is adapted to a product design task as an implementation example.

\section{Effective Retrieval Interaction For Task Performance Support - Example Of A Task Adaptive Manual For Filling Out A Form-}

For insurance contracts, workers must quickly refer to manuals and reply to the queries of insurance agencies and applicants. Employees require a great skill and task knowledge for the retrieval of proper items from a great volume of manual documents. For business performance support, traditionally there is a task manual that provides information and series of procedures for a task execution. To effectively execute a task, it is important to clearly represent the task procedure and 
to rapidly refer to the necessary manual contents based on task purpose and business procedure.

From the viewpoint of the human-machine interface of a task performance, the following issues must be considered for our task adaptive electronic manual:

1. Structuring and visualization of a task procedure (visual interface),

2. Task ontology (the explicit description of a task concept),

3. Transferring the performance skill of a task item,

4. A connective action for a relative task (the explicit representation of a definite task procedure).

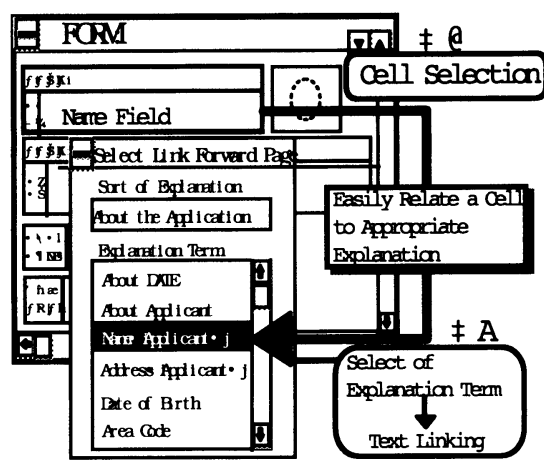

Authoring $\mathrm{I} / \mathrm{F}$

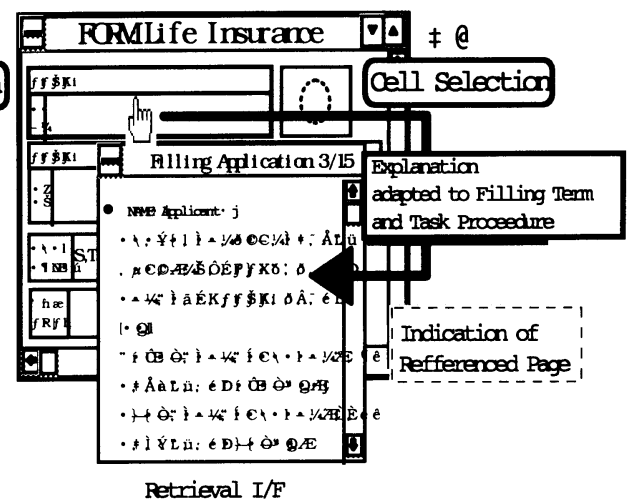

Retrieval I/F

For filling out a form, the following solutions are adopted to deal with these issues:

1. Using an application sheet structure in which each cell requires filling in,

2. A contract task is represented by an item's name in the form,

3. Storing and presenting an application form sample,

4. Automatic presentation of a related item from the contents of another manual.

This manual system for filling in an application form has a retrieval interface tool which enables referral to a suitable explanation in the manual for a task adaptation, and a hyper-link generating interface tool for easy content linking. Figure 1 shows a display example of link authoring and retrieval interface.

\section{Information Organizing System For Performance Support Of Products Design Task}

As mentioned above, it is effective for retrieval interaction to present an explicit description of a task procedure. In the product design field, design task knowledge, including design know-how or documents, is not always used to arrange or systematize, like manual contents because of production cycle, etc.. On the other hand, in joint products development with a collaborative design process formed in some project style, it is needed to support the gathering and organizing of 
distributed design information along with a common product design concept, purpose and work progress for related design workers. It is needed to quickly retrieve the design task information adapted from a large number design documents for design specifications, etc..

\subsection{Task performance support using an information organizing method}

Usually design information is stored in a database, and it is arranged according to each purpose of information creation. The retrieval of design information is divided into the following three cases as the results of a design task analysis:

1. the direct retrieval of a corresponding database with a known location of retrieval documents,

2. the selective retrieval of some database with a rough idea of the location of retrieval documents,

3. the overall retrieval of all related databases completely without an idea of the location of retrieval document.

Design workers are required in the three retrieval situations to rapidly retrieve some necessary document from many database files which are composed in different schemes. For this requirement, we propose the task support method using meta-data which organizes of relevant information adapted to the task.

Figure 2 shows the concept of task performance support system which use an information organizing and sharing method. In this system, the information searcher has selectively gathered material using relevant words defined by a task terminology dictionary. Meta-data describes gathering data which is mutually connected using the task model defined procedures. Meta-data refers to knowledge for the retrieval of design task information. Meta-knowledge, which is composed of the above mentioned task model and related data gathering, is stored in a common database, and results in viewpoint sharing of the internal and external company information.

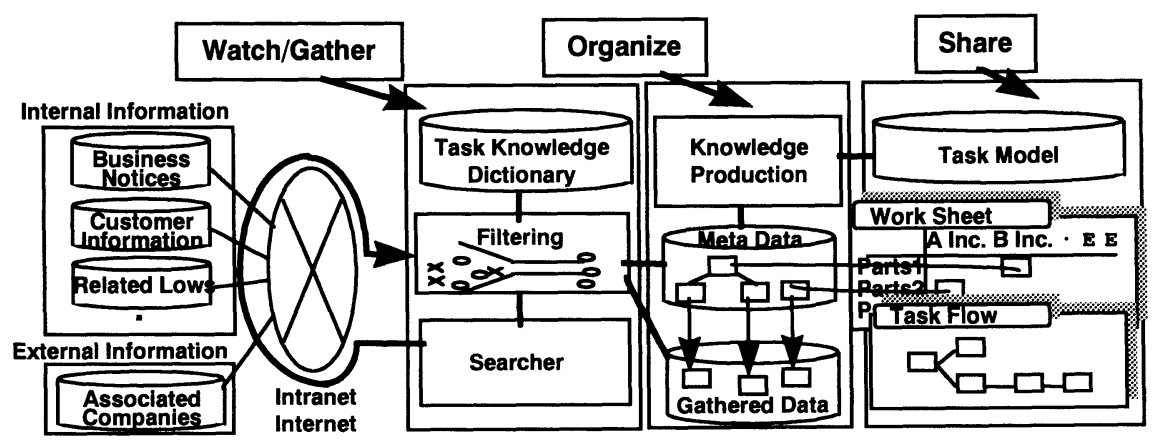

Figure 2. Basic Concept of Information Organizing and Sharing System 


\subsection{Information organization and retrieval using a task model}

In task execution which includes a creating process such as a design task, it is important to present workers with structured information along with a problem solution procedure for a task goal (i.e. task model). This is often needed to retrieve information including documents for parts selection, design memorandums, mail logs, minutes of proceedings, design drawings as well as the relating to this information. However, they usually use normal retrieval tools only. Actually workers must create a retrieval strategy which includes the selection of information sources and keywords in order to obtain useful information for their work. Additionally, the scope of information sources can be so broad that they cannot obtain retrieval results related to their work unless they repeat the retrieval process many times.

In order to solve the above mentioned problem, we use "task modeling" in organizing information for retrieval. The task modeling is based on the concept of "task ontology" (Mizoguchi,1994), and task models are defined and expressed in a form of tree structure or a form of table structure relating to some words of the task item.

A task model is used for an input key of the retrieval process. A piece of metaknowledge is made by retrieving keywords that are included in the task model, and embedding links that direct the retrieval results into the task model. We developed a prototype system which enables design workers to share contents (such as knowhow), create meta-knowledge with a task model and retrieve information related to task items. The information circulation and distribution image using this system is shown in Figure 3.

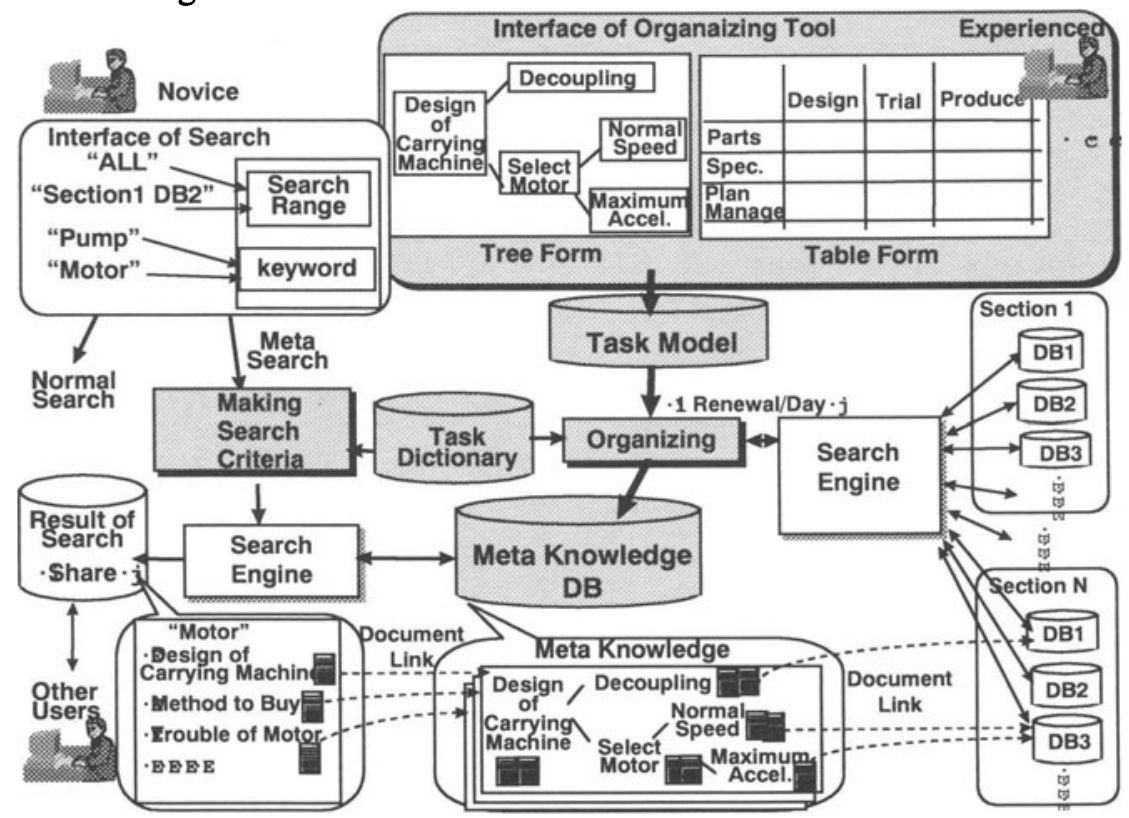

Figure 3. Implementation Image of Information Organizing and Sharing system 


\section{Conclusion}

We have developed an adaptive electronic manual system for filling out a form for an insurance contract. In this system, the form and guidance for the process are related by Hyper-Link. The proposed user interface enable rapid guidance retrieval by indicating a cell of filling form. This retrieval method with explicit task description is much effective for task performance in the same way as typical manual contents.

For distributed design task information, we proposed a method for design task performance support using the information organizing and sharing system. This system has the following functions and is expected to support the design task performance.

1. The experienced designers are able to easily make meta-knowledge for task know-how transferring by using a task model definition interface tool.

2. Beginners are able to obtain organized information in accordance with a task purpose with a few easy operations by retrieving the meta-knowledge created and stored by an experienced user.

\section{References}

Bramer, W.L. et al., 1994, Future Work. The Free Press.

Haga, H. et al., 1993, On the Multimedia Computer Aided Instruction System for Novice Programmers with Exercise Support Facility. IFIP Trans. A Vol.A-35. Kojima, H., 1992, Self-learning Electronic Manual using Multimedia-Basic Concept-. In: IPSJ(Japan), 45th Conf. Proc. B-4.

Mizoguchi, R., 1994, Current State of the Art of Knowledge Sharing and Reuse. In: Jour. of JSAI(Japan), 9(1).

Nonaka, I. et al., 1995, Knowledge Creating Company. Oxford University Press.

Yamada, T. et al., 1997, Human Interface Design Methodology for Electronic Manual System and its Application. In: 6th IEEE Int. Conf. on ETFA'97.

\section{Biography}

H. Kojima is a Senior Researcher of Systems Development Laboratory (S.D.L). He is engaged in the study of business performance and training support system and interactive information retrieval system. He holds a Ph.D. in Computer Application.

T. Yamada is a Researcher of S.D.L. He is engaged in the study of electronic manual system (E. M. System) and community ware system.

Y.Mizuno is Researcher of S.D.L. He is engaged in the development of E.M.System.

T. Yuasa is a Researcher of S.D.L. He is engaged in the development of task performance and training support system. 